\title{
Black and white holes at material junctions
}

\author{
Yaron Kedem, ${ }^{1}$ Emil J. Bergholtz $\odot,{ }^{1}$ and Frank Wilczek $\odot{ }^{1,2,3,4}$ \\ ${ }^{1}$ Department of Physics, Stockholm University, Stockholm SE-106 91 Sweden \\ ${ }^{2}$ Center for Theoretical Physics, Massachusetts Institute of Technology, Cambridge, Massachusetts 02139 USA \\ ${ }^{3}$ T. D. Lee Institute, and Wilczek Quantum Center, Department of Physics and Astronomy, Shanghai Jiao Tong University, \\ Shanghai 200240, China \\ ${ }^{4}$ Department of Physics and Origins Project, Arizona State University, Tempe Arizona 25287 USA
}

(Received 22 April 2020; accepted 27 October 2020; published 25 November 2020)

\begin{abstract}
Electrons in type II Weyl semimetals display one-way propagation, which supports totally reflecting behavior at an endpoint, as one has for black hole horizons viewed from the inside. Junctions of type I and type II lead to equations identical to what one has near black hole horizons, but the physical implications, we suggest, are quite different from expectations which are conventional in that context. The time-reversed, "white hole" configuration is also physically accessible.
\end{abstract}

DOI: 10.1103/PhysRevResearch.2.043285

There has been much interest in the simulation of black hole properties by laboratory systems [1-8]. Particularly intriguing, in this connection, is the possibility of realizing essentially quantum phenomena, notably including Hawking radiation [9] or the closely related Unruh effect [10]. In the laboratory systems physical space is essentially flat, but the equations of motion for some degrees of freedom resemble those for particles near a black hole event horizon. Systems under consideration include "sonic black holes" [11] embodied in Bose-Einstein condensates [2,8,12], optical systems $[3,13,14]$ or classical fluids $[15,16]$, and also inhomogeneous magnets [17], polaritons [18], thin films of ${ }^{3} \mathrm{He}-\mathrm{A}[19,20]$ and Weyl semimetals [21-25]. Here we will analyze different Weyl semimetal configurations which can mimic both black and white holes. We motivate idealized model Hamiltonians for these systems. Our models have interesting features, which, however, do not correspond to conventional expectations for black holes. In particular, the horizon has distinctive local properties, and there is no Hawking radiation.

Black and white hole geometries. A Weyl semimetal is a material in which the low energy description of the electronic band structure is given by the Hamiltonian $H= \pm \vec{\sigma} \cdot \vec{p}$, where $\vec{\sigma}$ is the vector of Pauli matrices pertaining to a band index, or spin, $\vec{p}$ is the momentum. For simplicity we set the Fermi velocity to unity. The two signs refer to two chiralities, which generally are located in different places in the Brillouin zone. Weyl semimetals were observed experimentally in tantalum arsenide (TaAs) [26-28] and later in a plethora of different materials [29]. Shortly after, "type II"

Published by the American Physical Society under the terms of the Creative Commons Attribution 4.0 International license. Further distribution of this work must maintain attribution to the author(s) and the published article's title, journal citation, and DOI. Funded by Bibsam.
Weyl semimetals [30], with over-tilted cones [31-34], were experimentally observed in, e.g., in molybdenum ditelluride $\left(\mathrm{MoTe}_{2}\right)$ [35], tungsten ditelluride $\left(\mathrm{WTe}_{2}\right)$ [36], and in TaIrTe [37]. Additional types were also considered [38]. Moreover, similar dispersion relations feature in metamaterials such as photonic crystals [39] and coupled waveguides [40].

Since condensed matter systems do not have Lorentz symmetry, an extra contribution to the Hamiltonian of the form $H_{\text {tilt }}=\vec{\kappa} \cdot \vec{p}$ can appear, where $\vec{\kappa}$ is a parameter depending on the details of the material [31]. These details might constrain $\vec{\kappa}$ to have some special form but here we consider a more general $\vec{\kappa}$. This term "tilts" the Weyl cone. For $|\vec{\kappa}|>1$ we have a type II dispersion with an overtipped cone [30], and there is only one direction of propagation. Space-dependent tilting of light cones is of course suggestive of black hole space-times. $\kappa$ can become space-dependent, for example, around the interface between Weyl semimetals of types I and II. To explore this analogy mathematically, we consider the Lagrangian density

$$
\mathcal{L}=i \bar{\psi}\left(\gamma^{\mu} \partial_{\mu}+\gamma^{0} \vec{\kappa}(\vec{x}) \cdot \vec{\partial}\right) \psi+\text { c.c. },
$$

where $\psi$ is a Dirac spinor [41] and $\gamma^{\mu}$ are the Dirac matrices. We observe that indeed the Lagrangian density in Eq. (1), which describes quasiparticles in a material, is the same as the one for a massless Dirac field in a curved space-time with the metric

$$
d s^{2}=\left(|\vec{\kappa}|^{2}-1\right) d t^{2}-2 \vec{\kappa} \cdot \overrightarrow{d x} d t+\overrightarrow{d x} \cdot \overrightarrow{d x} .
$$

In this sense, there is an event horizon at $|\vec{\kappa}|=1$.

This suggests that a condensed matter system which is described by the Lagrangian in Eq. (1) might supply an experimentally accessible vehicle to explore behaviors suggested for black holes, notably including Hawking radiation. On the other hand, that possibility raises serious issues. For example, there is no obvious energy source to power continued radiation. Before suggesting how this dilemma can be resolved, let us expand the context of the discussion, by considering the 
TABLE I. Interface configuration for type I and type II Weyl semimetal.

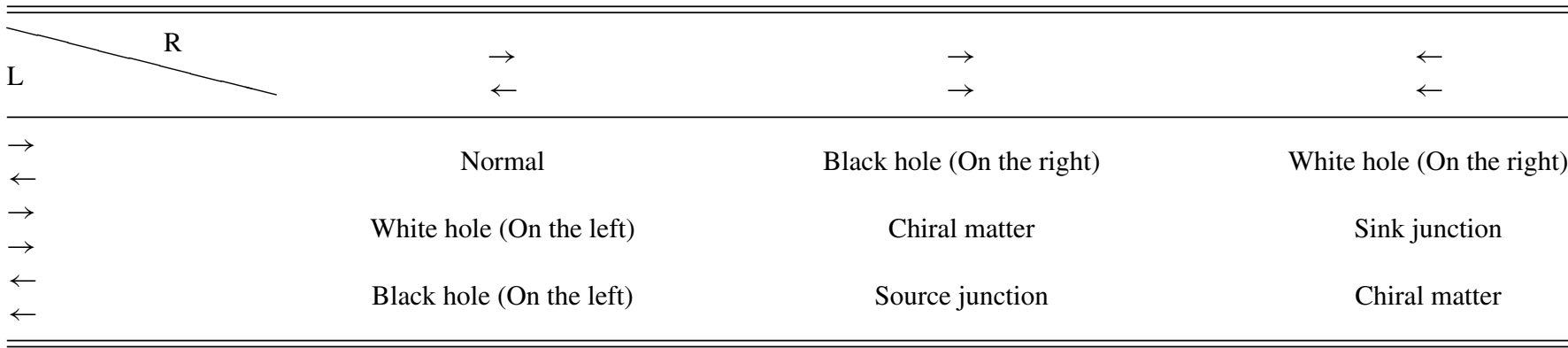

full range of possible "horizon" realizations. To keep things simple, by default we will specialize to one spatial dimension $z$. As will appear, the incorporation of other additional dimensions can bring in new physical possibilities, which deserve further investigation.

The overtilted cones imply modes that can propagate only in one direction. Consider first an interface between a region with two-way propagation and another where propagation is only possible away from the interface, making the interface totally repulsive. Since black holes are famous for their power of attraction, at first sight it may seem odd to associate a totally repulsive boundary with a black hole. But from the point of view of causal structure, the defining characteristic of a black hole horizon is that nothing escapes from the black hole side, interior (whereas its exterior supports both escape and capture). To achieve that feature, what is essential is that the horizon, viewed from the interior, is totally repulsive. Similarly, the defining feature of a white hole is that it is impossible to throw anything in, and that is achieved through total attraction, viewed from the inside. In Table I, we show the different possible configurations and their interpretations. It is also appropriate to note that the materials can terminate at "end of the world" boundaries, e.g., boundaries with empty space.

Wave packets and regularization. We can diagonalize $\gamma_{z}$ and analyze each component of $\psi$ separately. This corresponds to isolating definite chirality and spin. We have

$$
\mathcal{L}=i \psi^{*}\left[\partial_{t}+f(z) \partial_{z}\right] \psi+\text { c.c. },
$$

where $f(z)=\kappa(z) \pm 1$, with the sign depending on the particular component. The resulting Euler-Lagrange equation [42]

$$
\left[\partial_{t}+f(z) \partial_{z}+\frac{1}{2} \frac{\partial f}{\partial z}\right] \psi=0
$$

can be solved by the method of characteristics. Thus, we introduce a variable analogous to the tortoise coordinate of general relativity,

$$
r(z)=\int_{z_{0}}^{z} \frac{d u}{f(u)},
$$

with $z_{0} \neq 0$ chosen arbitrarily. Then we have, formally, the solution

$$
\psi(t, r)=\psi_{0}(r-t) \sqrt{\frac{|f(r-t)|}{|f(r)|}}
$$

in terms of a given wave form $\psi_{0}(r)$ at $t=0$. The subtlety here is that the coordinate $r(z)$ does not necessarily cover all values of $z$. Indeed, if $f(z)$ is positive for $z>0$ but approaches $z$ linearly for $z \rightarrow 0$, then the entire line $-\infty<r<\infty$ corresponds to the half-line $0<z<\infty$. If we consider wave packets $\psi_{0}$ supported on the half-line $0<z<\infty$, then there is no difficulty, and we find the simple qualitative behavior displayed in Fig. 1. Wave packets supported on the half-line $-\infty<z<0$ evolve analogously. It appears that the two sides behave independently. Note that we can also allow finite amplitude or even $1 / \sqrt{|f(z)|}$ singularities at $z=0$, so the evolution of all reasonable initial wave forms is covered.

To quantize the system and construct an appropriate (static) candidate ground state in a conventional way, we must work with energy eigenmodes. Note in this context that there is a preferred time for the electron field, which is set by the
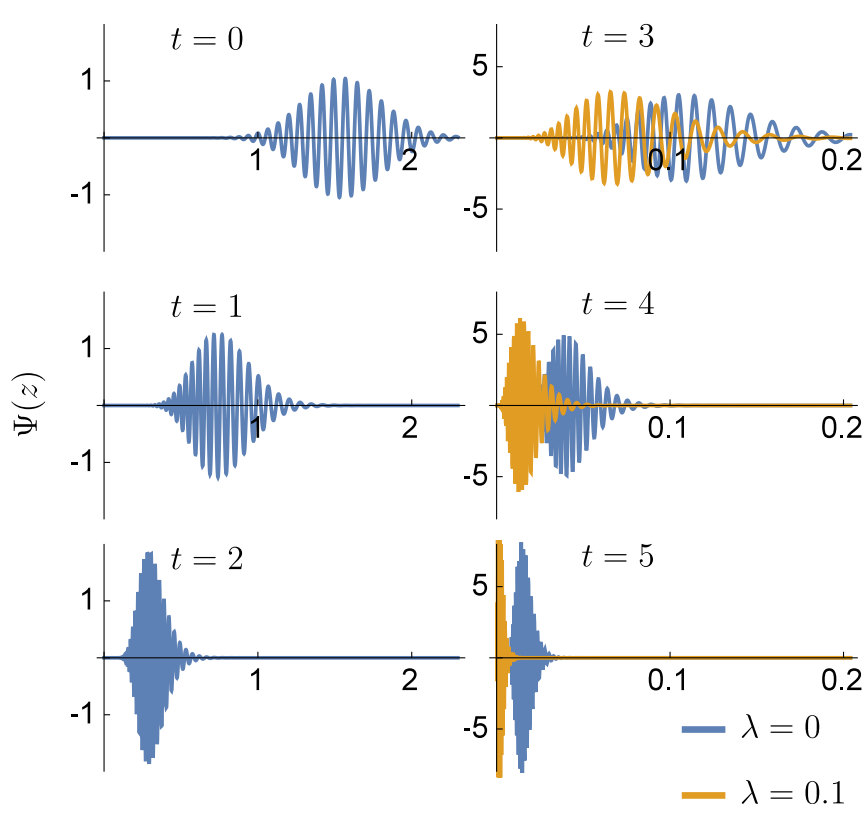

FIG. 1. The evolution of wave packets according to Eq. (6). Close to the horizon space is compressed, leading to distortion of the wave form. The local amplitude increases, due to the factor $\sqrt{|f(z)|}$ in the denominator, conserving the integrated density. The decreasing velocity ensures that wave packets supported away from $z=0$ never reach there. As discussed in the text, with regulators $\lambda>0$ the decrease is less rapid and the solution must be modified. The panel are labeled with timepoints to visualize a wave packet approaching a white hole horizon while alternative labels $t \rightarrow-t$ would correspond to a wave packet leaving a black hole horizon. 
external world and ultimately by the fact that after all our solid resides in flat space-time. More specifically, despite the metric's formal resemblance to black hole solutions of general relativity we need not consider alternative definitions of space-time inspired by the geometry defined by Eq. (2) on an equal footing, nor the "proper time" associated with electron trajectories. The Lagrangian Eq. (1) from which that geometry was derived is only an approximate model of highly restricted physical situations, and the background material which supports it has no simple generally covariant description.

Solutions of the kind

$$
\psi_{\omega}(t, z) \propto \frac{e^{-i \omega\left(t-\int^{z} \frac{d u}{f(u)}\right)}}{\sqrt{|f(z)|}},
$$

describe the eigenmodes. To use the modes in Eq. (7) in field quantization, we want them to form an orthonormal basis $\int d \omega \psi_{\omega}\left(z_{1}\right) \psi_{\omega}^{*}\left(z_{2}\right)=\delta\left(z_{1}-z_{2}\right), \int d z \psi_{\omega}(z) \psi_{\omega^{\prime}}^{*}(z)=$ $\delta\left(\omega-\omega^{\prime}\right)$. When $f(z)$ changes sign, say at $z=0$, the integral $\int_{z_{2}}^{z_{1}} \frac{d u}{f(u)}$ in the exponent can vanish for $z_{1} \neq z_{2}$ and modes localized at different positions would not be orthogonal. We resolve this issue by having separate modes in each region, $\psi_{\omega}^{L}(z)=\psi_{\omega}(z<0), \psi_{\omega}^{R}(z)=\psi_{\omega}(z>0)$. This separation was to be anticipated, in light of our preceding discussion of the initial value problem.

Another delicacy concerns normalization of the modes. One typically normalizes by putting the system in a finite sized box. Here, however, the effective horizon serves as one side of our box, and it is not entirely clear how to regulate logarithmically divergent normalization integral $\int d z|\psi|^{2} \propto$ $d z|f(z)|^{-1}$ (with $f(z) \propto z$ ). We can regulate this divergence, and arrive at an unambiguous proposal, by choosing a different form for $f(z)$ near the horizon, viz.

$$
f(z) \propto z \frac{(\lambda+|z|)^{\alpha}}{|z|^{\alpha}}
$$

where $\lambda \geqslant 0$ and $0<\alpha<1$ are parameters. This represents a steepening of the horizon onset. $\lambda$ sets the length scale for the regularization, while $\alpha$ determines the degree of steepening. Of course, many other similar regulators could be considered, including regulators which are not (anti)symmetric in $z \rightarrow-z$.

Inserting Eq. (8) into the metric of Eq. (2) with $f(z)=$ $\kappa(z) \pm 1$, we obtain a space-time with a lightlike singularity at $z=0$. If Eq. (2) is a solution to Einstein's equation a nonvanishing derivative of $\kappa(z)$ might imply a nonvanishing stress-energy tensor and the divergence of $\partial_{z} f(z)$ for Eq. (8), implies a diverging energy density. However, Einstein's equation does not describe the physics in our system and any related results or conjectures, such as the absence of naked singularities, - are irrelevant. This divergence represents the breakdown of the continuum limit at the horizon and does not affect the physics at distant regions. For simplicity of the presentation, we consider a continuous $\partial_{t}+f(z)$, but as since we have separate modes for each region, this is not essential to our result.

With $f(z)$ of the form Eq. (8), the tortoise coordinate $r(z)$ converges at the horizon, i.e., the half-line in $z$ maps onto a half-line in $r$. Following the general (formal) solution Eq. (6), we see that the wave packets now hit the horizon in a finite

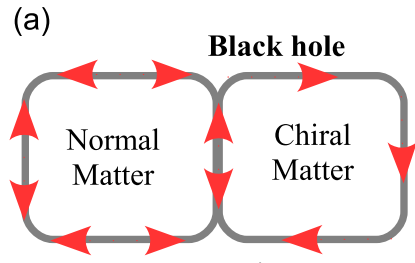

White hole

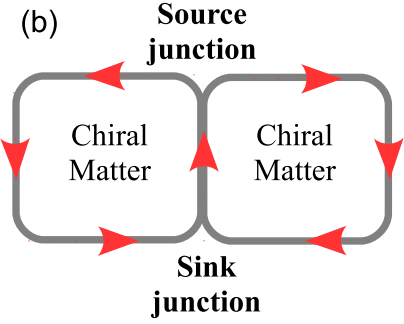

FIG. 2. Interfaces of chiral matter. The Lagrangian in Eq. (3) describes edge states of a chiral bulk, where the sign of $f(z)$ determines the chirality. The change of sign at the horizon is where edge states of two materials with different chiralities meet, with the interpretation given by Table I. The propagation along the interface is the analog of a wormhole connecting a black hole to a white one, while in the laboratory modes are simply moving in a different direction/dimension.

time. This, we argue, is a physically reasonable extrapolation of the suggested $\lambda=0$ behavior. Indeed, recall that in that limit we had wave packets accumulating at the horizon, yet never quite reaching it. But our solid has a natural minimum length scale, i.e., the lattice spacing, and once the packet is predicted to be squeezed below that scale, it should have arrived. That heuristic argument helps make the regulator appear physically reasonable, but it also foretells the breakdown of the formal solution Eq. (6), which is indeed valid only in the continuum limit. It is thus reasonable to consider such regulator when the naive continuum limit is invalid. To proceed further, we must extend the solution in a way consistent with physical requirements.

There are two possibilities which suggest themselves immediately, but do not withstand scrutiny. We might try to allow the wave packet to reflect back to where it came from. But this is impossible, because the propagation is unidirectional. Or, we might try to allow the wave packet to emerge on the opposite side of the horizon. But solutions on the other side propagate back toward the horizon, so no escape is possible. Thus it appears we must allow the wave packet to accumulate on the horizon, or to propagate elsewhere. The latter possibility can arise for chiral edge modes that bound higher-dimensional regions, as indicated in Fig. 2. The latter case can certainly arise in the context of the quantum Hall effect [43] and plausibly in other situations. Here, however, we shall pursue the former.

Model Hamiltonian and qualitative behavior. Allowing the wave packets to accumulate on the horizon suggest, in the context of quantization, that we should add a degree of freedom localized on the horizon which couples to the excitations described by the regularized eigenmodes. Let us consider how this can work. by

The Hamiltonian for the regularized eigenmodes is given

$$
H_{0}=\sum_{i=R, L} \int_{0}^{\infty} d \omega \omega\left(a_{i, \omega}^{\dagger} a_{i, \omega}+b_{i, \omega}^{\dagger} b_{i, \omega}\right),
$$

where $a_{i, \omega}^{(\dagger)}$ a (creation) annihilation operator for the mode $\psi_{\omega}^{i}$ and we relabeled $a_{i, \omega<0}^{\dagger} \rightarrow b_{i,-\omega}, a_{i, \omega<0} \rightarrow b_{i,-\omega}^{\dagger}$. The cou- 
pling to the localized degree of freedom is given by

$$
H_{I}=\sum_{i=R, L} \int_{-\infty}^{\infty} d \omega g_{i}(\omega)\left(A a_{i, \omega}^{\dagger}+A^{\dagger} a_{i, \omega}\right),
$$

where $A^{(\dagger)}$ is a (raising) lowering operator for the charge on the horizon, $Q=-A^{\dagger} A$, and $g_{i}(\omega)$ is a coupling function such that $g_{i}(z)=\int d \omega \psi_{\omega}^{i}(z) g_{i}(\omega)$ have significant support only close to the horizon. The energetic cost of charge accumulation is given by an onsite Hamiltonian $H_{c}=E_{c} Q^{2}$, with the charging energy $E_{c}$ depending on microscopic details.

Diagonalizing the complete Hamiltonian $H=H_{0}+H_{I}+$ $H_{c}$, the regularized eigenmodes are slightly modified, or dressed. Since the integral in Eq. (10) includes negative frequencies, we have terms such as $A^{\dagger} b_{i, \omega}^{\dagger}$ and $A b_{i, \omega}$. Thus if a particle reaching the horizon decreases the charge, a hole increases it. The complete Hamiltonian has a definite ground state, unlike the situation near a real black hole, which entail an instability. The stability of our model implies that there is no Hawking radiation.

Let us now look on the response of the system to a few types of external perturbations. First, consider applying a voltage across the system, i.e., running a current. We start with the case of a white hole. The external potential will create positively charged excitations, i.e., holes, on one side and negatively charged ones on the other. Both excitations will propagate towards the interface, canceling each others charge. To the experimentalist measuring the current it would simply look as if charge has moved from one side to the other. (Microscopic details might lead to a finite resistance or to local intermediate charge accumulation, which is the case in any inhomogeneous system). The case of a black hole is analogous, but the excitations are created at the interface and propagate to the two sides. This mechanism is reminiscent of the picture of Hawking radiation, based on pair creation $[44,45]$, but the details are quite different.

We can consider another scenario of placing some charge at the interface, say in a quenched way. In a type I Weyl semimetal, particle-hole pairs created by the Schwinger mechanism will screen the charge. A similar process occurs for type II as well. In contrast to the usual case, where the particle and the hole move in opposite direction due to the electric field, here both move in the same direction. However, as shown in Fig. 3, this phenomenon still allows for screening to occur.

An interesting feature of the causal structure emerges if we analyze the heat transfer in this system. Unlike charge for which an excitation can be negative or positive, all excitations imply adding energy to the system. In a system that has only left moving modes, heating a particular location will not affect anything to the right of that location. For any interface configuration, it is straightforward to see which region can be heated from any position and from which location one can measure any region. This is shown in Fig. 4. We see that the time-reversal exchange of black and white holes involves interchange of the excitation and measurement processes.

Summary and discussion. We have demonstrated that models of fermion propagation which arise as simple idealizations of behavior in type II Weyl semimetals and their junctions map onto models of fermion propagation in space-times with unusual causal structures: black holes, white holes, universal

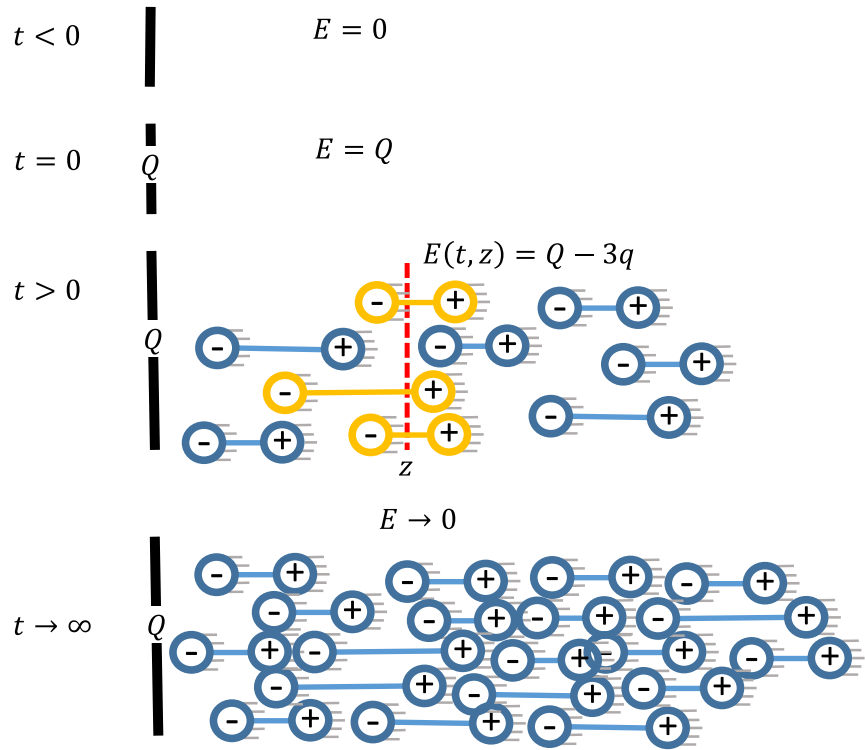

FIG. 3. Screening in type II Weyl semimetals of charge $Q$ placed at the interface (horizon) denoted by the black thick line. The pairs created by the Schwinger mechanism will propagate in one direction, conversely to the standard scenario where the particles propagate to opposite sides. As long as the electric field persist more pairs will be created. The momentary field at some position $z$, denoted by the red dashed line, is diminished by the number of pairs across that position. The steady state is a polarized bulk with vanishing electric field.

sources and universal sinks. We analyzed the behavior of wave packets and eigenmodes in those contexts, suggested how the models can be quantized in a way that regulates the infinite propagation delays at the horizon, at the cost of introducing an explicit boundary degree of freedom. We analyzed some simple but characteristic situations qualitatively, to demonstrate the physical consistency of this modeling.

In realistic Weyl semimetals there are always additional states at the Fermi level, in addition to those described by the
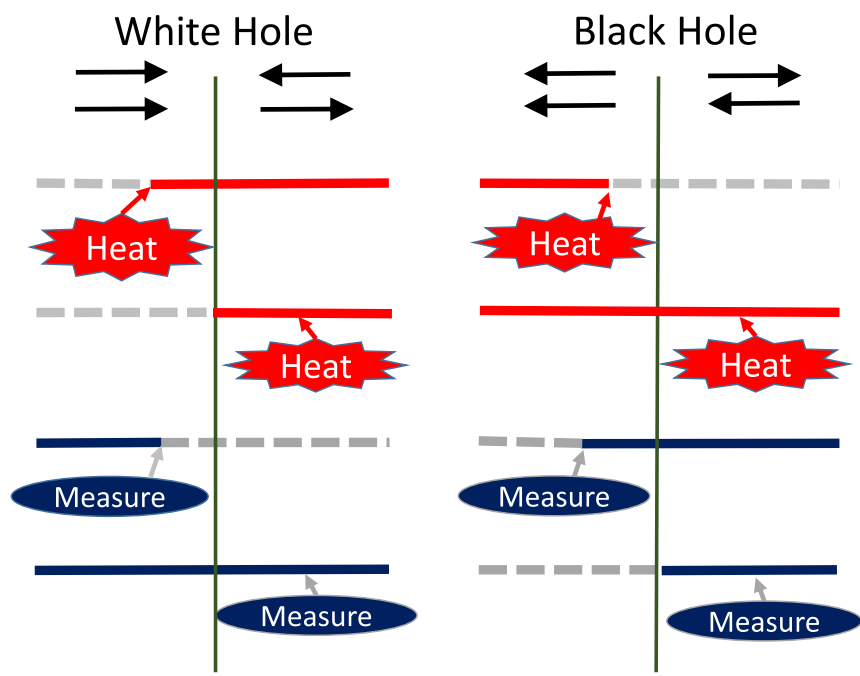

FIG. 4. Casual structure of an type I-type II Weyl semimetals interface. The colored region are accessible to the relevant operation, heating or measuring. Time reversal interchanges those operations. 
linearized low energy theory with a tilted Weyl cone. Most materials host parasitic bands that cross the Fermi level, and usually the Weyl cones occur at finite doping. Those are, however, not fundamental problems, and there are materials where they are avoided [46]. There are, however, also complications of a more fundamental nature: (i) The Nielsen Ninomiya theorem dictates that there are an even number of Weyl points in the Brillouin zone; (ii) The Weyl points are connected by Fermi arcs on the surface; (iii) The "light cones" are only approximate; to recover periodicity in the BZ the bands must curve back down (or up) away from the Weyl point. Those complications do not necessarily render the physical picture suggested by our linearized model of a single Weyl node with varying tilt irrelevant, because: (i) different Weyl nodes are essentially independent, at least in materials where they are well separated in momentum space; (ii) for appropriate surfaces, the Fermi arcs can be made small; (iii) band curvature, - e.g., adding mass introduces quantitative, but not qualitative alteration of the model. Prospects for engineering materials with the relevant properties as discussed in Ref. [47].

Although the starting equations were the same, the physical picture we have arrived at for our material system is quite different from conventional expectations for black holes. Among other things, our regularized horizon corresponds to a naked singularity, there is no Hawking radiation, and other types of exotic backgrounds (white holes, sources, sinks) appear on an equal footing with black holes. How did this happen, and what does it mean? The difference does not come from the microscopic details. Indeed the model in Eq. (1) describe the low energy, long wavelength physics and breaks at some scale (the same might be true for the Lagrangian of electrons as well), but this does not modify the arguments we have presented. The key point is that the equations of motion for some chosen degrees of freedom do not fully determine, independent of context, which solutions are physically relevant, nor how they should be interpreted. In our case, the existence of a preferred time and of a locally identifiable horizon are salient features of our material system which directly contradict standard, well-motivated assumptions about black holes. More generally, all proposed material analogs of black hole behavior require critical scrutiny, to determine how far the analogy can be taken. That said, consideration of material systems with unusual properties is valuable in itself, and might suggest new possibilities for the material system we call space-time.

Acknowledgments. We gratefully acknowledge useful discussions with S. Holst, T. Ojanen, and K. Zarembo. This work was supported by the Swedish Research Council under Contracts No. 335-2014-7424, No. 2016-04192_3, No. 201806720_3 and the Wallenberg Academy Fellows program of the Knut and Alice Wallenberg Foundation. In addition, F.W.'s work is supported by the U.S. Department of Energy under Contract No. DE-SC0012567 and by the European Research Council under Grant No. 742104.
[1] C. Barceló, S. Liberati, and M. Visser, Analog gravity, Living Rev. Relativity 14, 3 (2011).

[2] J. R. Muñoz de Nova, K. Golubkov, V. I. Kolobov, and J. Steinhauer, Observation of thermal Hawking radiation and its temperature in an analog black hole, Nature (London) 569, 688 (2019).

[3] J. Drori, Y. Rosenberg, D. Bermudez, Y. Silberberg, and U. Leonhardt, Observation of Stimulated Hawking Radiation in an Pptical Analog, Phys. Rev. Lett. 122, 010404 (2019).

[4] A. Fabbri and N. Pavloff, Momentum correlations as signature of sonic Hawking radiation in Bose-Einstein condensates, SciPost Phys. 4, 19 (2018).

[5] C. Sheng, H. Liu, Y. Wang, S. N. Zhu, and D. A. Genov, Trapping light by mimicking gravitational lensing, Nat. Photonics 7 , 902 (2013)

[6] R. Bekenstein, R. Schley, M. Mutzafi, C. Rotschild, and M. Segev, Optical simulations of gravitational effects in the Newton-Schrödinger system, Nat. Phys. 11, 872 (2015).

[7] U. Leonhardt, Questioning the recent observation of quantum Hawking radiation, Ann. Phys. 530, 1700114 (2018).

[8] Y.-H. Wang, T. Jacobson, M. Edwards, and C. W. Clark, Mechanism of stimulated Hawking radiation in a laboratory Bose-Einstein condensate, Phys. Rev. A 96, 023616 (2017).

[9] S. W. Hawking, Particle creation by black holes, Commun. Math. Phys. 43, 199 (1975).

[10] W. G. Unruh, Notes on black-hole evaporation, Phys. Rev. D 14, 870 (1976).

[11] W. G. Unruh, Experimental Black-Hole Evaporation? Phys. Rev. Lett. 46, 1351 (1981).
[12] J. Steinhauer, Observation of quantum Hawking radiation and its entanglement in an analog black hole, Nat. Phys. 12, 959 (2016).

[13] J. L. Gaona-Reyes and D. Bermudez, The theory of optical black hole lasers, Ann. Phys. 380, 41 (2017).

[14] T. G. Philbin, C. Kuklewicz, S. Robertson, S. Hill, F. König, and U. Leonhardt, Fiber-optical analog of the event horizon, Science 319, 1367 (2008).

[15] S. Weinfurtner, E. W. Tedford, M. C. J. Penrice, W. G. Unruh, and G. A. Lawrence, Measurement of Stimulated Hawking Emission in an Analog System, Phys. Rev. Lett. 106, 021302 (2011).

[16] L.-P. Euvé, F. Michel, R. Parentani, T. G. Philbin, and G. Rousseaux, Observation of Noise Correlated by the Hawking Effect in a Water Tank, Phys. Rev. Lett. 117, 121301 (2016).

[17] A. Roldán-Molina, A. S. Nunez, and R. A. Duine, Magnonic Black Holes, Phys. Rev. Lett. 118, 061301 (2017).

[18] H. S. Nguyen, D. Gerace, I. Carusotto, D. Sanvitto, E Galopin, A. Lemaître, I. Sagnes, J. Bloch, and A. Amo, Acoustic Black Hole in a Stationary Hydrodynamic Flow of Microcavity Polaritons, Phys. Rev. Lett. 114, 036402 (2015).

[19] T. Jacobson and T. Koike, Black hole and baby universe in a thin film of 3 he-a, in Artificial Black Holes (World Scientific, Singapore, 2002), pp. 87-108.

[20] T. A. Jacobson and G. E. Volovik, Effective spacetime and Hawking radiation from a moving domain wall in a thin film of 3 he-a, J. Exp. Theor. Phys. Lett. 68, 874 (1998). 
[21] G. E. Volovik, Black hole and Hawking radiation by type-ii Weyl fermions, JETP Lett. 104, 645 (2016).

[22] H. Huang, K.-H. Jin, and F. Liu, Black-hole horizon in the dirac semimetal $\mathrm{zn}_{2} \mathrm{in}_{2} \mathrm{~s}_{5}$, Phys. Rev. B 98, 121110(R) (2018).

[23] L. Liang and T. Ojanen, Curved spacetime theory of inhomogeneous Weyl materials, Phys. Rev. Research 1, 032006(R) (2019).

[24] S. Guan, Z.-M. Yu, Y. Liu, G.-B. Liu, L. Dong, Y. Lu, Y. Yao, and S. A. Yang, Artificial gravity field, astrophysical analogues, and topological phase transitions in strained topological semimetals, npj Quantum Mater. 2, 23 (2017).

[25] H. Liu, J.-T. Sun, H. Huang, F. Liu, and S. Meng, Fermionic analog of black hole radiation with a super high Hawking temperature, arXiv:1809.00479 (2018).

[26] S.-Y. Xu, I. Belopolski, N. Alidoust, M. Neupane, G. Bian, C. Zhang, R. Sankar, G. Chang, Z. Yuan, C.-C. Lee, S.-M. Huang, H. Zheng, J. Ma, D. S. Sanchez, B. Wang, A. Bansil, F. Chou, P. P. Shibayev, H. Lin, S. Jia, and M. Z. Hasan, Discovery of a Weyl fermion semimetal and topological fermi arcs, Science 349, 613 (2015).

[27] B. Q. Lv, N. Xu, H. M. Weng, J. Z. Ma, P. Richard, X. C. Huang, L. X. Zhao, G. F. Chen, C. E. Matt, F. Bisti et al., Observation of Weyl nodes in taas, Nat. Phys. 11, 724 (2015).

[28] S.-M. Huang, S.-Y. Xu, I. Belopolski, C.-C. Lee, G. Chang, B. Wang, N. Alidoust, G. Bian, M. Neupane, C. Zhang, et $a l$., A Weyl fermion semimetal with surface fermi arcs in the transition metal monopnictide taas class, Nat. Commun. 6, 7373 (2015).

[29] N. P. Armitage, E. J. Mele, and A. Vishwanath, Weyl and dirac semimetals in three-dimensional solids, Rev. Mod. Phys. 90, 015001 (2018).

[30] A. A. Soluyanov, D. Gresch, Z. Wang, Q. Wu, M. Troyer, X. Dai, and B. A. Bernevig, Type-II Weyl semimetals, Nature (London) 527, 495 (2015).

[31] E. J. Bergholtz, Z. Liu, M. Trescher, R. Moessner, and M. Udagawa, Topology and Interactions in a Frustrated Slab: Tuning from Weyl Semimetals to $\mathcal{C}>1$ Fractional Chern Insulators, Phys. Rev. Lett. 114, 016806 (2015).

[32] Y. Xu, F. Zhang, and C. Zhang, Structured Weyl Points in Spin-Orbit Coupled Fermionic Superfluids, Phys. Rev. Lett. 115, 265304 (2015).

[33] M. Trescher, B. Sbierski, P. W. Brouwer, and E. J. Bergholtz, Quantum transport in dirac materials: Signatures of tilted and anisotropic Dirac and Weyl cones, Phys. Rev. B 91, 115135 (2015).
[34] A. Kobayashi, S. Katayama, Y. Suzumura, and H. Fukuyama, Massless fermions in organic conductor, J. Phys. Soc. Jpn. 76, 034711 (2007).

[35] K. Deng, G. Wan, P. Deng, K. Zhang, S. Ding, E. Wang, M. Yan, H. Huang, H. Zhang, Z. Xu et al., Experimental observation of topological fermi arcs in type-II Weyl semimetal mote 2, Nat. Phys. 12, 1105 (2016).

[36] C. Wang, Y. Zhang, J. Huang, S. Nie, G. Liu, A. Liang, Y. Zhang, B. Shen, J. Liu, C. Hu, Y. Ding, D. Liu, Y. Hu, S. He, L. Zhao, L. Yu, J. Hu, J. Wei, Z. Mao, Y. Shi, X. Jia, F. Zhang, S. Zhang, F. Yang, Z. Wang, Q. Peng, H. Weng, X. Dai, Z. Fang, Z. Xu, C. Chen, and X. J. Zhou, Observation of Fermi arc and its connection with bulk states in the candidate type-II Weyl

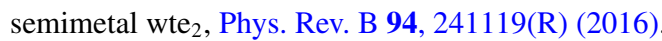

[37] E. Haubold, K. Koepernik, D. Efremov, S. Khim, A. Fedorov, Y. Kushnirenko, J. van den Brink, S. Wurmehl, B. Büchner, T. K. Kim, M. Hoesch, K. Sumida, K. Taguchi, T. Yoshikawa, A. Kimura, T. Okuda, and S. V. Borisenko, Experimental realization of type-II Weyl state in noncentrosymmetric tairte ${ }_{4}$, Phys. Rev. B 95, 241108(R) (2017).

[38] J. Nissinen and G. E. Volovik, Type-III and IV interacting Weyl points, JETP Lett. 105, 447 (2017).

[39] L. Lu, Z. Wang, D. Ye, L. Ran, L. Fu, J. D. Joannopoulos, and M. Soljačić, Experimental observation of Weyl points, Science 349, 622 (2015) ..

[40] J. Noh, S. Huang, D. Leykam, Y. D. Chong, K. P. Chen, and M. C. Rechtsman, Experimental observation of optical Weyl points and Fermi arc-like surface states, Nat. Phys. 13, 611 (2017).

[41] The Hamiltonian for a tilted Weyl semimetal, $H+H_{\text {tilt }}=(\vec{\kappa} \pm$ $\vec{\sigma}) \cdot \vec{p}$, corresponds to two of the four components of $\psi$.

[42] One can obtain the third term in Eq. (4) from the Hermitian form of the tilting Hamiltonian $H_{\text {tilt }}=[\vec{\kappa}(\vec{x}) \cdot \vec{p}+\vec{p} \cdot \vec{\kappa}(\vec{x})] / 2$.

[43] M. Stone, An analog of hawking radiation in the quantum Hall effect, Classical and quantum gravity 30, 085003 (2013).

[44] Don N. Page, Particle emission rates from a black hole: Massless particles from an uncharged, nonrotating hole, Phys. Rev. D 13, 198 (1976).

[45] Maulik K. Parikh and F. Wilczek, Hawking Radiation as Tunneling, Phys. Rev. Lett. 85, 5042 (2000).

[46] Z. Wang, M. G. Vergniory, S. Kushwaha, M. Hirschberger, E. V. Chulkov, A. Ernst, N. P. Ong, R. J. Cava, and B. A. Bernevig, Time-Reversal-Breaking Weyl Fermions in Magnetic Heusler Alloys, Phys. Rev. Lett. 117, 236401 (2016).

[47] A. A. Burkov and L. Balents, Weyl Semimetal in a Topological Insulator Multilayer, Phys. Rev. Lett. 107, 127205 (2011). 